\title{
TOXICITE D'UN DERIVE BI-AZOTE, L'HYDRATE D'HYDRAZINE POUR CARASSIUS CARASSIUS L. ET RUTILUS RUTILUS L. ET DIFFERENTS STADES DU DEVELOPPEMENT DU BRACHYDANIO RERIO H.B.
}

\author{
par J. P. PROTEAU, P. LIM et R. LABAT ${ }^{1}$
}

La toxicité de l'hydrate d'hydrazine, dérivé bi-azoté, est étudiée chez deux poissons d'eau douce tempérée, Carassius carassius L. et Rutilus rutilus L., et un poisson d'eau douce tropicale, Brachydanio rerio H.B., par des tests de létalité tet de sublétalité.

L'hydrate d'hydrazine se révèle plus toxique pour Carassius carassius $\left(\mathrm{CL}_{50-} 24 \mathrm{~h}\right.$ à $\left.19^{\circ} \mathrm{C} \pm 1=1,480 \mathrm{mg} / \mathrm{l}\right)$ et Rutilus rutilus $\left(\mathrm{CL}_{50^{-}} 24 \mathrm{~h}\right.$ à $\left.19^{\circ} \mathrm{C} \pm 1=0,850 \mathrm{mg} / \mathrm{l}\right)$ que pour Brachydanio rerio $\left(\mathrm{CL}_{30}-24\right.$ à $\left.20^{\circ} \mathrm{C} \pm 1=3,18 \mathrm{mg} / \mathrm{l}\right)$ retenu comme animal-test dans la norme AFNOR T 90303.

La toxicité de ce produit varie avec le stade du développement chez brachydanio rerio; elle est environ trois fois plus forte pour les alevins de 5 jours, que pour des pré-adultes ( 3 mois) ; enfin, l'exposition à des concentrations assez fortes (6 à $26,5 \mathrm{ppm})$ a provoqué des déformations visibles de la colonne vertébrale sur les embryons de ce poisson, sans toutefois provoquer de mortalité immédiate (24 heurcs) comme c'était le cas pour les autres stades du développement. L'hydrate d'hydrazine semble donc avoir un effet tératogène chez cette espèce.

\section{Toxicity of a nitrogenous derivative, hydrazine hydrate, for Carassius carassius L., Rutilus rutilus $L$. \\ and different developmental stages of Brachydanio rerio H. B.}

The toxicity of hydrazine hydrate, a nitrogenous derivative, was studied with two freshwater fish from the temperate zone, Carassius carassius L. and Rutilus rutilus L., and with one freshwater species from the tropics, Brachydanio rerio H. B., by tests of lethal and sub-lethal doses.

Hydrazine hydrate is more toxic for Carassius carassius $\left(\mathrm{CL}_{50}-24 \mathrm{~h}\right.$ at $19{ }^{\circ} \mathrm{C}$ $\left.\pm 1=1.480 \mathrm{mg}^{-1}\right)$ and Rutilus rutilus $\left(\mathrm{CL}_{\mathrm{BO}}-24 \mathrm{~h}\right.$ at $\left.19^{\circ} \mathrm{C} \pm 1=0.850 \mathrm{mg}^{-1}\right)$ than for Brachydanio rerio $\left(\mathrm{CL}_{\mathrm{so}}-24 \mathrm{~h}\right.$ at $\left.20^{\circ} \mathrm{C} \pm 1=3.18 \mathrm{mg} \mathrm{1^{-1 }}\right)$ kept as animaltest in the standard AFNOR T 90303.

The toxicity of this product varies with the developmental stage of $B$. rerio; it is about three times stronger for the alevins, about 5 days old, than for pre-adults ( 3 months) ; finally, high concentrations (6 to $26.5 \mathrm{ppm}$ ) produced visible deformations of the vertebral column in the embryos of the fish, without however an immediate mortality ( 24 hours), as was the case in the other stages of development. Hydrazine hydrate seems therefore to have a malforming effect on this species.

1. E.N.S. Agronomique, 145, avenue de Muret, 31076 Toulouse Cedex. 


\section{INTRODUCTION}

La pollution de certains cours d'eau en produits azotés (ammoniac, hydrazine...) nous amène à étudier la toxicité de ces produits vis-à-vis des poissons. Au scin des écosystèmes dulçaquicoles, les poissons représentent, en effet, le niveau ultime d'intégration des effets des pollutions, par leur étroite dépendance vis-à-vis des facteurs abiotiques de leur environnement et de la chaîne trophique; ils constituent de ce fait, un matériel biologique privilégié pour les études des pollutions aquatiques. Dans l'étude qui va suivre, nous nous intéresserons en particulier à l'hydrate d'hydrazine.

La mise en évidence de la toxicité des produits chimiques pour l'environnement soulève de nombreux problèmes inhérents à la complexité et à l'interaction de différents produits qui se trouvent dans le milieu. Le choix d'une méthode d'étude reposant sur la détermination des concentrations létales moyennes pendant une durée déterminée, ne reflète qu'une partie de ce qui se passe dans l'écosystème aquatique, mais néanmoins, il nous montre la relation concentration du produit-temps de survie, en fonction de la température et du $\mathrm{pH}$ du milieu étudié.

Pour comprendre l'action du toxique sur le cycle des poissons, des tests complémentaires s'imposent ; c'est ainsi que nous avons pu constater un effet tératogène de l'hydrate d'hydrazine sur les œufs de Brachydanio rerio en effectuant des tests de toxicité sur différents stades du développement de ce poisson.

\section{1. - MATERIEL ET METHODES}

L'hydrazine utilisée provient de P.C.U.K. Lannemezan, la synthèse du produit étant réalisée suivant la réaction :

$2 \mathrm{NH}_{3}+\mathrm{ClONa} \rightarrow \mathrm{ClNa}+\mathrm{N}_{2} \mathrm{H}_{4}, \mathrm{H}_{2} \mathrm{O}$ (p.m. $\left.=32,048\right)$.

Ce produit est employé dans l'industrie, comme agent phyto-pharmaceutique, base d'agent gonflant pour caoutchoucs et plastiques, traitement des eaux de chaudières et enfin, comme agent de synthèses chimiques et pharmaceutiques.

L'hydrazine est un produit très soluble dans l'eau et la transformation de l'hydrazine en hydrate $\left(\mathrm{N}_{2} \mathrm{H}_{4}, \mathrm{H}_{2} \mathrm{O} \rightarrow \mathrm{N}_{2} \mathrm{H}_{5}{ }^{+}+\mathrm{OH}^{-}\right)$la stabilise quelque peu : pour vérifier sa stabilité lors des tests effectués sur

1. P.O. Box 907, Ames, Iowa 50010, 515-232-2533 - TWX/Telex - 910-520-1158. 
les poissons, nous avons mesuré les concentrations du produit après plusieurs heures ( $7 \mathrm{~h} 30,14 \mathrm{~h} 30$ et 24 h) d'expérience (Cf. tableau I, expériences $\mathrm{n}^{\circ} 1$ et 2), le dosage étant réalisé dans tous les cas par colorimétrie à l'aide de l'Hydraver II préparé par Hach Chemical Company ${ }^{1}$. Il ressort de ces expériences que la dégradation de l'hydrazine est plus importante en milieu aéré $(32,4 \%$ à $58,2 \%$ contre 34 à $37 \%$ ) au bout de la durée de 24 heures ; l'hydrazine est en effet oxydée lentement par différents corps chimiques, en particulier par l'oxygène.

Tableau I. - Stabilité de l'hydrazine.

Expérience $n^{\circ} 1:$ sans aération artificielle $-t=20^{\circ} \mathrm{C} \pm 0,5$.

\begin{tabular}{|c|c|c|c|c|c|}
\hline & Expérience $\mathrm{n}^{\circ} 1$ & $1^{\text {er }}$ & aquarium & $2^{e}$ & quarium \\
\hline \multirow{5}{*}{$t=0$} & Volume (1) & & 4 & & 4 \\
\hline & $\left(\mathrm{N}_{2} \mathrm{H}_{4}, \mathrm{H}_{2} \mathrm{O}\right)-\mathrm{mg} / \mathrm{l}$ & & 1,30 & & 0,60 \\
\hline & $\mathrm{pH}$ & & 8,01 & & 8,00 \\
\hline & Résistivité $(\mathrm{k} \Omega$ ) & & 1,61 & & 1,60 \\
\hline & Oxygène dissous - mg/1 & 9 & $(102 \%)$ & 9 & $(102 \%)$ \\
\hline \multirow{3}{*}{$t=7 \mathrm{~h} 30$} & $\left(\mathrm{~N}_{2} \mathrm{H}_{4}, \mathrm{H}_{2} \mathrm{O}\right)-\mathrm{mg} / \mathrm{l}$ & 1,13 & $(86,9 \%)$ & 0,53 & $(88,3 \%)$ \\
\hline & $\mathrm{pH}$ & \multicolumn{2}{|r|}{7,95} & \multicolumn{2}{|r|}{7,90} \\
\hline & Oxygène dissous - mg/l & 7,3 & $(82,5 \%)$ & 7,4 & $(83,7 \%)$ \\
\hline \multirow{4}{*}{$t=24 h$} & $\left(\mathrm{~N}_{2} \mathrm{H}_{4}, \mathrm{H}_{3} \mathrm{O}\right)-\mathrm{mg} / \mathrm{l}$ & 0,82 & $(63 \%)$ & 0,40 & $(66 \%)$ \\
\hline & pH & \multicolumn{2}{|r|}{7,95} & \multicolumn{2}{|r|}{7,89} \\
\hline & Résistivité $(\mathrm{k} \Omega)$ & \multicolumn{2}{|r|}{1,60} & \multicolumn{2}{|r|}{1,61} \\
\hline & Oxygène dissous - mg/1 & 6,6 & $(74,6 \%)$ & 6,55 & $(74,1 \%)$ \\
\hline
\end{tabular}

Expérience $n^{\circ} 2$ : avec aération artificielle $-t=20^{\circ} \mathrm{C} \pm 0,5$.

\begin{tabular}{|c|c|c|c|c|c|c|c|}
\hline & Expérience $\mathrm{n}^{\circ} 2$ & $1^{\text {er }}$ a & aquarium & $2^{e}$ ac & quarium & $3^{e}$ ac & quarium \\
\hline & Volume (l) & & 22 & & 22 & & 22 \\
\hline & $\left(\mathrm{N}_{2} \mathrm{H}_{4}, \mathrm{H}_{2} \mathrm{O}\right)-\mathrm{mg} / \mathrm{l}$ & & 0,260 & & 0,550 & & 1,05 \\
\hline \multirow[t]{4}{*}{$t=0$} & $\mathrm{pH}$ & & 7,9 & & 7,9 & & 7,9 \\
\hline & Résistivité $(\mathrm{k} \Omega)$ & & 1,62 & & 1,62 & & 1,62 \\
\hline & Oxygène dissous - $\mathrm{mg} / \mathrm{l}$ & 6 & $(67,8 \%)$ & 5,6 & $(63 \%)$ & 5,6 & $(63 \%)$ \\
\hline & $\left(\mathrm{N}_{2} \mathrm{H}_{4}, \mathrm{H}_{2} \mathrm{O}\right)-\mathrm{mg} / \mathrm{l}$ & 0,14 & $4(54 \%)$ & 0,325 & $5\left(\begin{array}{ll}59 & \%\end{array}\right)$ & 0,675 & $(64 \%)$ \\
\hline \multirow[t]{3}{*}{$t=14 \mathrm{~h} 30$} & $\mathrm{pH}$ & & 7,9 & & 7,9 & & 7,9 \\
\hline & Résistivité $(\mathrm{k} \Omega)$ & & 1,61 & & 1,61 & & 1,61 \\
\hline & $\left(\mathrm{N}_{2} \mathrm{H}_{4}, \mathrm{H}_{2} \mathrm{O}\right)-\mathrm{mg} / \mathrm{l}$ & 0,11 & $1(42,3 \%)$ & 0,23 & $(41,8 \%)$ & 0,50 & $(47,6 \%)$ \\
\hline \multirow[t]{3}{*}{$t=24 h$} & $\mathrm{pH}$ & & 7,9 & & 7,9 & & 7,9 \\
\hline & Résistivité $(\mathrm{k} \Omega)$ & & 1,61 & & 1,62 & & 1,62 \\
\hline & Oxygène dissous $-\mathrm{mg} / \mathrm{l}$ & 7,6 & $(86 \%)$ & 7,8 & $(88,2 \%)$ & 8 & $(90,5 \%)$ \\
\hline
\end{tabular}


Les stades pré-adultes de trois espèces dulçaquicoles ont été retenus pour les tests :

- Carassius carassius L. (Carassin), poisson d'eau tempérée,

- Rutilus rutilus L. (Gardon), poisson d'eau tempérée,

- Brachydanio rerio H.B. (Poisson zèbre), poisson d'eau chaude.

Chez ce dernier, deux stades supplémentaires du développement (œufs et alevins) ont été testés. Les tests sur les pré-adultes et sur les alevins sont réalisés sur des lots homogènes de poissons soumis à différentes concentrations d'hydrazine après 15 jours d'acclimatation au milieu de dilution. La température est maintenue constante $\left(19^{\circ} \mathrm{C}\right.$ \pm 1 pour Carassius carassius et Rutilus rutilus; $20^{\circ} \mathrm{C} \pm 1$ pour $\mathrm{Bra}$ chydanio rerio; $26^{\circ} \mathrm{C} \pm 1$ pour les alevins) pendant toute la durée de l'expérience. Pour chaque espèce, le nombre de poissons morts aux diverses concentrations est noté après 24 heures d'exposition à l'hydrazine et le calcul de la $\mathrm{CL}_{50}$ est effectué après transformation log-probit (Finney, 1952) et pondération quand cela est possible.

L'eau de dilution était de l'eau de ville pour Carassius carassius et Rutilus rutilus et de l'eau synthétique préparée selon les normes AFNOR pour Brachydanio rerio. La composition chimique de ces milieux figure au tableau II. Pour Carassius carassius et Rutilus rutilus, les tests ont porté sur quatre concentrations comprises respectivement entre 0,5 et $1,2 \mathrm{mg} / 1$ et 0,9 et $2,5 \mathrm{mg} / 1$, les sujets ayant un poids moyen de $8 \mathrm{~g}$. Le $\mathrm{pH}$ des solutions s'est maintenu entre 8,1 et 8,5 et l'oxygène en fin d'expérience est resté supérieur à $6 \mathrm{mg} / \mathrm{l}$.

Tableau II. - Eaux expérimentales.

\begin{tabular}{|c|c|c|}
\hline & Eau de ville & Eau synthétique \\
\hline Température $\left({ }^{\circ} \mathrm{C}\right)$ & 25 & $\ldots \ldots$ \\
\hline Résistivité $\mathrm{k} \Omega . \mathrm{Cm}$ & 3,4 & N.D. \\
\hline $\mathrm{pH}$ & 7,8 & $7,8 \pm 0,1$ \\
\hline $\begin{array}{ll}\mathrm{Ca}^{++} & \mathrm{mg} / \mathrm{l} \mathrm{CaCO} \\
\mathrm{mg} / \mathrm{C} \mathrm{Ca}^{++}\end{array}$ & $\begin{array}{r}100 \\
40\end{array}$ & $\begin{array}{r}110 \\
44\end{array}$ \\
\hline $\begin{array}{ll}\mathrm{Mg}^{++} & \begin{array}{l}\mathrm{mg} / \mathrm{I} \mathrm{CaCO} \\
\mathrm{mg} / 1 \mathrm{Mg}^{++}\end{array}\end{array}$ & $\begin{array}{l}35 \\
8,4\end{array}$ & N.D. \\
\hline T.A.C. $\begin{array}{l}\mathrm{mg} / \mathrm{l} \mathrm{CaCO} \\
\mathrm{mg} / 1 \mathrm{HCO}_{3}^{-}\end{array}$ & $\begin{array}{r}110 \\
67\end{array}$ & N.D. \\
\hline $\mathrm{Cl}^{-} \quad \mathrm{mg} / \mathrm{l} \mathrm{Cl}{ }^{-}$ & 15 & 64 \\
\hline $\mathrm{SO}_{4}=\mathrm{mg} / \mathrm{S} \mathrm{SO}_{4}=$ & 16 & N.D. \\
\hline $\mathrm{PO}_{3}^{-}{ }_{4} \mathrm{mg} / \mathrm{A} \mathrm{PO}^{3-}$ & 0,04 & N.D. \\
\hline
\end{tabular}

Pour les stades pré-adultes de Brachydanio rerio, d'une taille moyenne de $3,3 \mathrm{~cm}$, nous avons testé sept concentrations différentes allant de 1,48 à $7,14 \mathrm{mg} / 1$. Chez les alevins de cette espèce, cinq concen- 
Tableau III. - Toxicité de l'hydrazine pour Rutilus rutilus et Carassius carassius.

Rutilus rutilus $\mathrm{L}$.

\begin{tabular}{cccc} 
Nombre de sujets testés & $\begin{array}{c}\mathrm{x} \\
\mathrm{(C)}\end{array} \mathrm{mg} / \mathbf{1}$ & $\begin{array}{c}\mathrm{y} \\
\text { Nombre de morts }\end{array}$ & $\begin{array}{c}\% \\
\text { de mortalité }\end{array}$ \\
\hline 5 & 0,5 & 0 & 0 \\
5 & 0.7 & 0 & 0 \\
5 & 0.9 & 3 & 60 \\
5 & 1,2 & 5 & 100 \\
\hline
\end{tabular}

Carassius carassius $\mathbf{L}$.
$x$
y
$\%$

Nombre de sujets testés

(C) $\mathrm{mg} / \mathrm{l} \quad$ Nombre de morts

de mortalité

\begin{tabular}{rrrr}
\hline 5 & 0.9 & 0 & 0 \\
5 & 1.2 & 1 & 20 \\
5 & 1.7 & 3 & 60 \\
5 & 2.5 & 5 & 100 \\
\hline
\end{tabular}

trations $(0,7$ à $2,45 \mathrm{mg} / 1)$ ont été testées. L'évolution du $\mathrm{pH}$, de l'oxygène dissous et des concentrations en hydrazine pendant l'expérience figure dans les tableaux IV (pré-adultes) et V (alevins). Enfin, deux tests ont été réalisés sur des œufs embryonnés soumis à différentes concentrations d'hydrazine pendant 96 heures (expérience I) et 120 heures (expérience II).

TableaU IV. - Toxicité de l'hydrazine pour les pré-adultes de Brachydanio rerio.

\section{Test préliminaire}

$$
\mathrm{C}(\mathrm{mg} / \mathrm{l})
$$

$\begin{array}{cccccc}0,001 & 0,01 & 0,1 & 1 & 10 & \begin{array}{c}0 \\ \text { (témoin) } \\ 5\end{array} \\ 5 & 5 & 5 & 5 & 5 \\ 0 & 0 & 0 & 0 & 5 & 0\end{array}$

Nombre de poissons introduits

Nombre de poissons morts après 24 heures

Test définitif

$$
\text { C (mg/l) }
$$

Nb. poissons introduits

Nb. poissons morts après 24 heures

$\%$ mortalité

O. fin d'expérience (\% saturation) $\left(\mathrm{N}_{2} \mathrm{H}_{1}, \mathrm{H}_{2} \mathrm{O}\right)-\mathrm{mg} / \mathrm{l}$ $\mathrm{pH}$

$\begin{array}{cccccccc}\begin{array}{c}0 \\ \begin{array}{c}\text { (Témoin) } \\ 10\end{array}\end{array} & 10 & 10 & 10 & 10 & 10 & 10 & 10 \\ & & & & & & & \\ 0 & 0 & 1 & 3 & 5 & 8 & 9 & 10 \\ 0 & 0 & 10 & 30 & 50 & 80 & 90 & 100 \\ & & & & & & & \\ 85 & 76 & 74,6 & 74,1 & 77 & 73,4 & 73,2 & 73 \\ 0 & 0,94 & 1,26 & 1,53 & 1,95 & 2,49 & 3,13 & 4,21 \\ 7,6 & 7,7 & 7,6 & 7,8 & 8 & 8,1 & 8,0 & 8,2\end{array}$


TableaU V. - Toxicité de l'hydrazine pour les alevins de Brachydanio rerio (alevins do 5 jours).

\begin{tabular}{lcccccc}
\hline \multicolumn{1}{c}{$C(\mathrm{mg} / \mathrm{l})$} & Témoin & 0,70 & 0,90 & 1,40 & 1,75 & 2,45 \\
\hline $\begin{array}{l}\text { Nombre poissons } \\
\text { introciuits }\end{array}$ & 10 & 10 & 10 & 10 & 10 & 10 \\
O. (\% saturation) & 100 & 98 & 100 & $>100$ & $>100$ & 100 \\
$\begin{array}{l}\text { Nb. poissons morts } \\
\quad \text { après 24 heures }\end{array}$ & 0 & 1 & 3 & 7 & 8 & 9 \\
\% mortalité & 0 & 10 & 30 & 70 & 80 & 90 \\
$\mathrm{O}_{\text {, fin (\% saturation) }}$ & 84 & 75,6 & 742 & 81,5 & 72,4 & 71,9 \\
$\left(\mathrm{~N} . \mathrm{H}_{\mathbf{s}}\right)$ & 0 & 0,55 & 0,66 & 1,09 & 1,27 & 1,76 \\
\hline
\end{tabular}

\section{2. - RESULTATS}

\subsection{Toxicité aiguë}

Pour Rutilus rutilus, le calcul de la $\mathrm{CL}_{50} .24 \mathrm{~h}$ à partir des résultats (Tableau III) donne $0,85 \mathrm{mg} / 1 \pm 0,37$ alors que pour Carassius carassius la $\mathrm{CL}_{50-} 24 \mathrm{~h}$ est de $1,48 \mathrm{mg} / \mathrm{l} \pm 0,56$ à la même température de $10^{\circ} \mathrm{C} \pm 1$ (Tableau VI).

Tableau VI. - Résultats des calculs des $\mathrm{CL}_{5,1}-24 \mathrm{~h}$ de l'hydrazine.

\begin{tabular}{lcccc}
\hline Espèces & Rutilus rutilus & $\begin{array}{c}\text { Carassius } \\
\text { carassius }\end{array}$ & $\begin{array}{c}\text { Brachydanio } \\
\text { rerio } \\
\text { pré-adulte }\end{array}$ & $\begin{array}{c}\text { Brachydanio } \\
\text { rerio } \\
\text { alevin 5 jours }\end{array}$ \\
\hline $\mathrm{CL}_{53} 24 \mathrm{~h}$ & 0,85 & 1,48 & 3,18 & 1,17 \\
I.C. $95 \%$ & 0,37 & 0,56 & 0,64 & 0,48 \\
T ${ }^{\circ} \mathrm{C}$ & $19^{\circ} \mathrm{C} \pm 1$ & $19^{\circ} \mathrm{C} \pm 1$ & $20^{\circ} \mathrm{C} \pm 1$ & $26^{\circ} \mathrm{C} \pm 0,5$ \\
\hline
\end{tabular}

Chez Brachdanio rerio, le calcul de la $\mathrm{CL}_{\text {, w }}$. $24 \mathrm{~h}$ de l'hydrazine à partir des résultats (Tableaux IV et V) donne $3,18 \mathrm{mg} / \mathrm{l} \pm 0,64 \mathrm{chez}$ les pré-adultes à $20^{\circ} \mathrm{C}$ contre $1,17 \mathrm{mg} / \pm 0,48 \mathrm{chez}$ les alevins âgés de 5 jours ̀̀ $26^{\circ} \mathrm{C}$ (Tableau VI).

\subsection{Toxicité sublétale}

Au cours de la première expérience (Tableau VII), la mortalité des œufs témoins atteint $40 \%$ en 96 heures alors que les œufs traités à l'hydrazine ne sont ni opaques, ni transparents comme les témoins; le cœur des embryons traités $(96 \mathrm{~h})$ bat très lentement. Aucune éclosion n'est observée en 96 heures chez les traités alors qu'elle a eu lieu normalement chez les témoins. 
TableaU VII. - Toxicité de l'hydrazine pour les œufs du Brachydanio rerio.

$\omega_{\mathrm{m}}=$ œufs morts; al.m = alevins morts ; $\Sigma_{\mathrm{m}}=$ total des morts ; $\% \mathrm{mc}=\%$ mortalité cumulée; $\% \mathrm{mcc}=\%$ mortalité cumulée corrigée.

Nombre d'œufs introduits par bac: 10 .

\begin{tabular}{|c|c|c|c|c|c|c|}
\hline \multicolumn{7}{|c|}{ Expérience I } \\
\hline & $\mathrm{C}(\mathrm{mg} / \mathrm{l})$ & $\begin{array}{c}0 \\
\text { (témoin) }\end{array}$ & 12 & 18 & 23 & 26,5 \\
\hline $\mathbf{t}=\mathbf{0}$ & Nombre introduit & 10 & 10 & 10 & 10 & 10 \\
\hline $\mathrm{t}=24 \mathrm{~h}$ & Nombre d'œufs anormaux & 0 & 4 & 5 & 8 & 10 \\
\hline \multirow[t]{2}{*}{$\mathrm{t}=96 \mathrm{~h}$} & Nombre morts & 4 & - & - & - & - \\
\hline & $\left(\mathrm{N}_{2} \mathrm{H}_{4}\right)-\mathrm{mg} / \mathrm{l}$ & 0 & 9,18 & 14,07 & 17,50 & 17,03 \\
\hline
\end{tabular}

Expérience II

$$
\text { Témoin } \quad \mathrm{C}_{1}=6 \mathrm{ppm} \quad \mathrm{C}_{2}=7,8 \mathrm{ppm}
$$

\begin{tabular}{|c|c|c|c|c|c|c|c|c|c|c|c|c|c|c|c|}
\hline & \multicolumn{5}{|c|}{ Témoin } & \multicolumn{5}{|c|}{$\mathrm{C}_{1}=6 \mathrm{ppm}$} & \multicolumn{5}{|c|}{$\mathrm{C}_{2}=7,8 \mathrm{ppm}$} \\
\hline & $\omega_{m}$ & al.m & $\Sigma_{m}$ & & $\% \mathrm{mcc}$ & $\omega_{m}$ & al.m & $\Sigma_{\mathrm{m}}$ & & $\% \mathrm{mcc}$ & $\omega_{\mathrm{m}}$ & al.m & $\Sigma_{m}$ & $\% \mathrm{mc}$ & $\% \mathrm{mcc}$ \\
\hline $\mathrm{t}=24 \mathrm{~h}$ & 3 & - & 3 & 30 & 0 & 1 & - & 1 & 10 & 0 & 0 & - & 0 & 0 & 0 \\
\hline$t=72 \mathrm{~h}$ & - & - & 0 & 30 & 0 & 1 & - & 1 & 20 & 0 & 0 & - & 0 & 0 & 0 \\
\hline $\mathrm{t}=120 \mathrm{~h}$ & - & - & 0 & 30 & 0 & 0 & 10 & 10 & 100 & 100 & 7 & 3 & 10 & 100 & 100 \\
\hline \multirow[t]{3}{*}{$\begin{array}{c}\left(\mathrm{N}_{2} \mathrm{H}_{4}\right) \\
\mathrm{mg} / \mathrm{l}\end{array}$} & \multicolumn{5}{|c|}{0} & \multicolumn{5}{|c|}{2,28} & \multicolumn{5}{|c|}{3,20} \\
\hline & \multicolumn{5}{|c|}{$C_{3}=10,1 \mathrm{ppm}$} & \multicolumn{5}{|c|}{$\mathrm{C}_{4}=13,2 \mathrm{ppm}$} & \multicolumn{5}{|c|}{$C_{3}=17,9 \mathrm{ppm}$} \\
\hline & $\omega_{\mathrm{m}}$ & al.m & $\Sigma_{m}$ & $\% \mathrm{mc}$ & $\% \mathrm{mcc}$ & $\omega_{\mathrm{m}}$ & al.m & $\Sigma_{11}$ & $\% \mathrm{mc}$ & $\% \mathrm{mcc}$ & $\omega_{m n}$ & al.m & $\Sigma_{m}$ & $\% \mathrm{mc}$ & $\% \mathrm{mcc}$ \\
\hline$t=24 \mathrm{~h}$ & 0 & - & 0 & 0 & 0 & 0 & - & 0 & 0 & 0 & 0 & - & 0 & 0 & 0 \\
\hline$t=72 \mathrm{~h}$ & 0 & - & 0 & 0 & 0 & 1 & - & 1 & 10 & 0 & 1 & - & 1 & 10 & 0 \\
\hline $\mathrm{t}=120 \mathrm{~h}$ & 7 & 3 & 10 & 100 & 100 & 7 & 3 & 10 & 100 & 100 & 8 & 2 & 10 & 100 & 100 \\
\hline $\begin{array}{c}\left(\mathrm{N}_{2} \mathrm{H}_{4}\right) \\
\mathrm{mg} / \mathrm{l}\end{array}$ & \multicolumn{5}{|c|}{3,64} & \multicolumn{5}{|c|}{5,44} & \multicolumn{5}{|c|}{6,27} \\
\hline
\end{tabular}

En revanche, l'examen des embryons traités au microscope optique ( $\times 36)$ révèle l'existence de déformations au niveau de la colonne vertébrale et un sac vitellin anormalement opaque. De plus, les embryons gardent la position qu'ils avaient dans l'œuf lorsqu'on déchire la membrane externe de celui-ci (fig. 1) et demeurent rigides, le cœur battant toujours. Ces malformations sont décelables 24 heures après le traite. ment à l'hydrazine, avec une plus grande fréquence de cas dans les fortes concentrations. Après 96 heures, ces malformations atteignent l'ensemble des œufs dans les différentes contractions sans qu'il soit possible, dans ces conditions d'observation, d'établir un gradient des effets en fonction des concentrations.

Lors de la seconde expérience (Tableau VII), nous n'avons pas observé de mortalité significative en 72 heures d'expérience. les té- 
moins étant les plus affectés (30\% de mortalité dès 24 heures). Cent vingt heures après le cébut de l'expérience, et un jour après l'éclosion théorique, la mortalité est totale chez les traités, dans toutes les concentrations. Il a pu éclore un certain nombre d'œufs, d'autant plus nombreux que la concentration est plus faible, mais les alevins meurent tous.

Les alevins ou les embryons traités par les différentes concentrations d'hydrazine présentent tous des malformations de la colonne vertébrale, semblables à celles décrites lors de l'expérience $\mathrm{n}^{\circ} 1$ et ce, même clans la plus faible concentration (fig. 1).
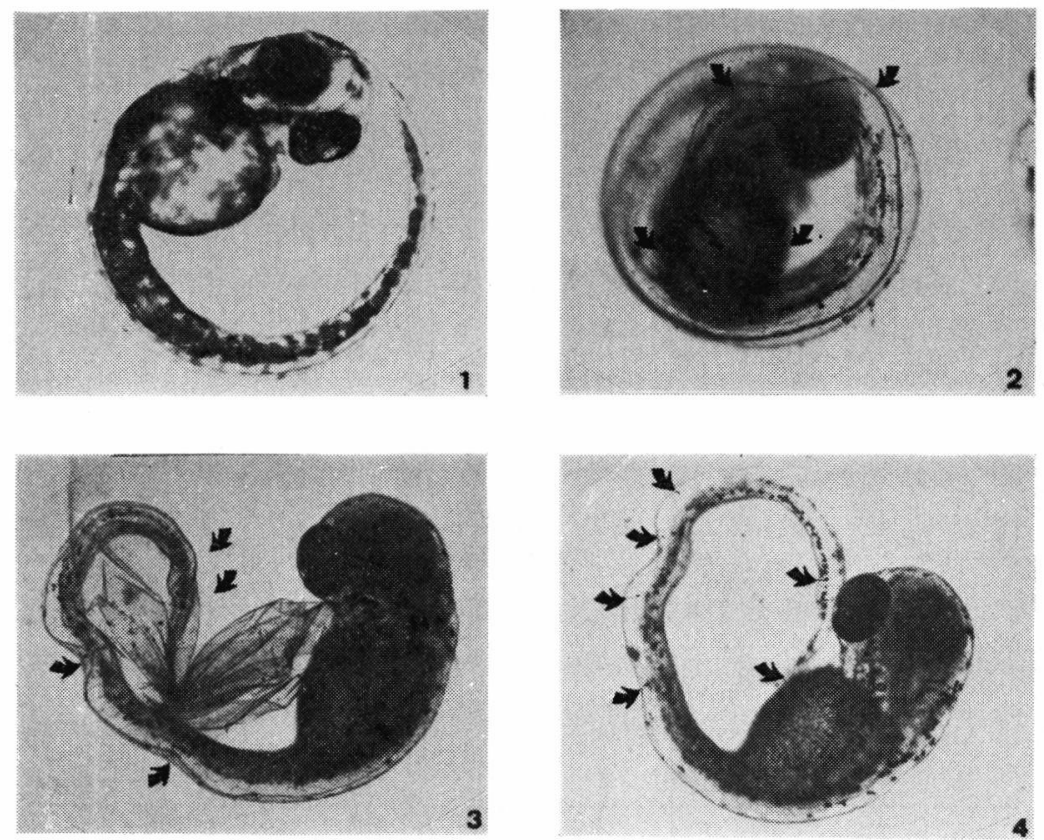

Fis. 1. - Effets de l'hydrazine sur le développement embryonnaire du Brachydanio rerio H.B.

Taille réelle de l'œuf: $1 \mathrm{~mm}$; grossissement $\times 36$ (microscope optiqus). 1: Stade XXIII témoin ; 2 : Même stade; hydrazine $\mathrm{C}=12 \mathrm{ppm} ; 3$ : Même œuf; membrane déchirée ; 4 : Même œuf, une heure plus tard.

\section{3. - DISCUSSION ET CONCLUSIONS}

Dans des conditions expérimentales identiques et avec des poissons de même taille, la $\mathrm{CL}_{50^{-}} 24 \mathrm{~h}$ de l'ammoniaque est de 8,6 ppm $\pm 0,84$ pour Rutilus rutilus et de 17,25 ppm $\pm 3,21$ pour Carassius carassius (Lim 1977) ; l'hydrazine, dans des conditions expérimentales identiques, est donc, pour ces poissons, 10 à 11 fois plus toxique que l'ammoniaque (étude comparative de toxicité entre $\mathrm{NH}_{4}$ et $\mathrm{N}_{2} \mathrm{H}_{4}$ en cours). 
Les pré-adultes de Gardons et de Carassins, poissons d'eau tempérée, sont plus sensibles à l'action de l'hydrazine que le Brachydanio rerio H.B. $\left(\mathrm{CL}_{50} \cdot 24 \mathrm{~h}=3,49 \mathrm{ppm} \pm 0,705\right)$ poisson d'eau chaude retenu comme animal-test par l'AFNOR; il faut souligner d'autre part que les deux premières espèces ont une sensibilité voisine à l'action de l'hydrazine ( $\mathrm{CL}_{50}$ - $24 \mathrm{~h}$ Rutilus rutilus: $0,85 \mathrm{ppm} \pm 0,37$ et $\mathrm{CL}_{50}$. 24 h Carassius carassius: 1,48 ppm $\pm 0,56$ ), ce qui n'était pas le cas avec l'ammoniaque.

Le stade du développement peut influer sur la sensibilité aux toxiques; ainsi, les jeunes alevins de 5 jours du Brachydanio rerio sont trois fois plus sensibles (si l'on néglige l'influence de la température : $20^{\circ} \mathrm{C}$ et $26^{\circ} \mathrm{C}$ ) à l'action de l'hydrazine que les pré-adultes $\left(\mathrm{CL}_{50(1)} .24 \mathrm{~h}\right.$ pré-aduites $=3,49 \mathrm{ppm} \pm 0,70 ; \mathrm{CL}_{5,-} 24 \mathrm{~h}$ alevins de 5 jours $=$ $1,17 \mathrm{ppm} \pm 0,48)$, alors qu'une concentration aussi forte que $26,5 \mathrm{ppm}$ n'a pas provoqué de mortalité en 24 heures chez les embryons traités.

Pour Huet (1960), la perméabilité des téguments aux substances toxiques serait plus grande chez les alevins vésiculés; la sensibilité importante des alevins pourrait trouver son origine dans ce phénomène. En effet, Skidmore (1965) a montré que ce sont les alevins fraîchement éclos (4 à 13 jours) qui survivent le moins longtemps à diverses concentrations de zinc, alors que la sensibilité d'alevins de 7 jours du même Brachydanio rerio est, pour le bichromate de potassium, voisine de celle des pré-adultes: 264,3 à 345,1 ppm pour les alevins de 7 jours contre 213,1 à 267,1 pour les pré-adultes (Proteau 1978). Il est donc difficile de conclure, d'une manière générale, que la sensibilité des jeunes alevins, est plus grande que celle des autres stades du développement du Brachydanio rerio; la nature du toxique semble jouer un rôle important dans cette sensibilité.

Matériel biologique délicat, les œufs sont cependant relativement résistants à l'action du bichromate de potassium, de l'hydrazine et du sulfate de zinc (Skidmore 1965), ce qui ne permet pas leur emploi pour l'évaluation des $\mathrm{CL}_{\overline{5}()} .24 \mathrm{~h}$ sur ce poisson. Cependant, la détection des substances tératogènes peut être effectuée avec profit sur ces œufs. Ainsi, l'hydrazine nous est apparue comme un produit à effet tératogène possible sur le Brachydanio rerio H.B. à des concentrations létales pour les pré-adultes. Skidmore (196j) a observé que le temps de survie des œufs décroît avec l'âge, jusqu'à l'éclosion, en présence de sulfate de zinc. Dans un second travail (1966), le même auteur a montré que la résistance de l'œuf cu Danio zébré ne provient pas de l'existence de la membrane externe, qui a même tendance à la diminuer pour les fortes concentrations de $\mathrm{ZnSO}_{4}$. Il constate alors la formation d'une zone opaque lorsque la membrane est présente et l'embryon meurt. L'origine de cette résistance demeure donc inconnue. 
D'autres travaux à venir devraient permettre d'éclaircir et de préciser l'action de ce produit, notamment à des concentrations plus faibles.

\section{TRAVAUX CITÉS}

A.F.N.O.R. (Association Française de Normalisation). - Documents du Comité d'Etude des Eaux et Norme T 90 303, juin 1978: détermination de la toxicité aiguë d'une substance vis-à-vis de Brachydanio rerio (essai statique), $4 \mathrm{p}$.

Barbier (P.), Leynaud (G.) et Champ (P.). 1973. - Les tests de toxicité sur les organismes aquatiques et leur interprétation statistique. C.T.G.R.E.F., rapport $\mathrm{n}^{\circ} 3,30 \mathrm{p}$.

FInNEY (D. J.). 1952 (réimpression 1962 et 1964). - Probit Analysis, 318 p. Cambridge University Press.

Hisaoka (K. K.) et BatTle (H. I.). 1958. - The normal developmental stages of the Zebrafish, Brachydanio rerio. J. Morphol., 102 : 311-328.

Huet (M.). 1950. - Toxicologie des poissons. Bulletin du Centre belge d'Etude et de Documentation des Eaux, $\mathrm{n}^{\circ} 7,1950 / 1$ : 396-406.

Lim (P.). 1977. - Etude de la dépollution de la Petite Baïse. Thèse Doct. Spéc., I.N.P.-E.N.S.A. Toulouse, 162 p.

Proteau (J.P.). 1978. - Recherches sur la toxicité de l'acide cyanurique et du phosphogypese au niveau des écosystèmes aquatiques. Thèse de $3^{*}$ cycle, I.N.P.-E.N.S.A. Toulouse, 250 p.

SKIDMORE (J. F.). 1965. - Resistance to zinc sulphate of the Zebrafish (Brachydanio rerio H.B.) at different phases of its life history. Ann. Appl. Biol., 56:47-53.

SKIDMore (J. F.). 1966. - Resistance to zinc sulphate of Zebrafish (Brachydanio rerio H.B.) embryos after removal or rupture of the outer egg membrane. J. Fish Res. Board of Canada, 23 (7) : 1037-1041.

Février 1980. 